\title{
Conventional and microwave-modulated Shubnikov-de Haas oscillations in GaN electron systems
}

\author{
J.R. Juanga , D.R. Hanga,b, Tsai-Yu Huang, W.K. Hung, , Y.F. Chen ${ }^{\mathrm{a}}$, Gil-Ho Kim ${ }^{\mathrm{d}}$, \\ Ming-Gu Lin ${ }^{\mathrm{a}}$, Tse-Ming Chen ${ }^{\mathrm{a}}$, C.-T. Liang ${ }^{\mathrm{a}, *}$, Y. Lee ${ }^{\mathrm{d}}$, Jae-Hoon Lee ${ }^{\mathrm{e}}$, \\ Jung-Hee Lee \\ ${ }^{a}$ Department of Physics, National Taiwan University, Taipei 106, Taiwan \\ ${ }^{\mathrm{b}}$ Department of Materials Science and Opto-Electronic Engineering, National Sun Yat-Sen University, Kaohsiung 804, Taiwan \\ ${ }^{\mathrm{c}}$ Department of Electro-optical Engineering, National Taipei University of Technology, Taipei, Taiwan \\ ${ }^{\mathrm{d}}$ Department of Electronic and Electrical Engineering, Sungkyunkwan University, Suwon 440-760, South Korea \\ ${ }^{\mathrm{e}}$ School of Electronic and Electrical Engineering, Kyungpook National University, Taegu 702-701, South Korea
}

\begin{abstract}
We report the drastic enhancement of Shubnikov-de Haas ( $\mathrm{SdH}$ ) oscillations observed in a GaN/AlGaN heterostructure by microwave modulation. The dependence of the $\mathrm{SdH}$ pattern on microwave power and temperature are investigated. The underlying mechanism is attributed to the effect of carrier heating. This technique has the advantage of keeping the carrier concentration fixed and not requiring expensive high-energy laser facilities compared with carrier-modulated $\mathrm{SdH}$ measurements. Moreover, we have investigated the low-temperature transport properties of front-gated $\mathrm{Al}_{0.18} \mathrm{Ga}_{0.82} \mathrm{~N} / \mathrm{GaN}$ heterostructures. By changing the applied gate voltage, we can vary the carrier density in our sample. At high carrier densities ( $n>4.65 \times 10^{12} \mathrm{~cm}^{-2}$ ), the measured mobility $(\mu)$ is found to be a decreasing function of carrier density as $\mu \sim n^{-0.31}$. Loss of mobility with increasing carrier density is dominated by interface roughness scattering. At low carrier densities $\left(n<4.24 \times 10^{12} \mathrm{~cm}^{-2}\right.$ ), the measured mobility is found to be an increasing function of carrier density as $\mu \sim n^{0.34}$. This is consistent with remote ionized impurity scattering, although the measured exponent 0.34 is smaller than the typical value (0.7-1.5) observed in an $\mathrm{AlGaN} / \mathrm{GaN}$ electron system. A possible reason is that our sample mobility is approximately five times lower than those in other devices for a similar electron density.
\end{abstract}

(C) 2003 Elsevier B.V. All rights reserved.

PACS: 73.61.- r

Keywords: GaN; Shubnikov-de Haas; Microwave

Recent efforts in developing III-V nitride family, $\mathrm{InN}, \mathrm{GaN}$, and $\mathrm{AlN}$ have led to significant progress in improving material quality. Alloys and heterostructures based on these materials are therefore being

\footnotetext{
* Corresponding author. Tel.: +886-223-697238; fax: +886223-63998.

E-mail address: ctliang@phys.ntu.edu.tw (C.-T. Liang).
}

studied with great interest. A high-quality $2 \mathrm{DEG}$ can form in the GaN/AlGaN heterointerface because of the large band offset and the strong piezoelectric and spontaneous polarization in this material system. We know that observation of the Shubnikov-de Haas $(\mathrm{SdH})$ oscillations requires that the thermal energy and the scattering-induced energy broadening be smaller than the Landau level separation. Therefore, 
the $\mathrm{SdH}$ technique is not suitable for the measurement at high temperatures or for samples with relatively low mobility. Many experimental techniques have been developed to enhance the sensitivity of the conventional $\mathrm{SdH}$ measurements. For example, the carrier-modulated $\mathrm{SdH}$ technique [1,2], which is based on measuring the changes in magnetoresistance by a chopped laser light source. However, because the additional laser source can generate excess carriers, it is very difficult to obtain the exact carrier concentration as well as to determine the underlying mechanism of the enhanced SdH pattern.

In this paper, we present measurements of microwave-modulated and conventional $\mathrm{SdH}$ oscillations in $\mathrm{GaN} / \mathrm{AlGaN}$ electron systems. Our experimental results fall into two categories. In the first part of the paper, we present a novel technique that can greatly enhance the $\mathrm{SdH}$ pattern without changing the carrier concentration. The enhanced $\mathrm{SdH}$ pattern is obtained by recording the changes in the quantum oscillations of magnetoresistance due to microwave radiation. We demonstrate that this technique is suitable for studying the magnetoresistance at relatively high temperature and in samples with moderate mobilities. In the second part of the paper, we report magnetotransport measurements on a gated $\mathrm{GaN} / \mathrm{Al}_{0.18} \mathrm{Ga}_{0.82} \mathrm{~N}$ electron system. We will investigate both the carrier density dependence of the electron mobility in our system.

In the first part, the measurements were performed on a metalorganic chemical vapor deposition (MOCVD) grown $\mathrm{GaN} / \mathrm{Al}_{0.4} \mathrm{Ga}_{0.6} \mathrm{~N}$ heterostructure. The sample was grown on a sapphire substrate and consists of an undoped $250 \AA$ thick $\mathrm{Al}_{0.4} \mathrm{Ga}_{0.6} \mathrm{~N}$, an undoped $\mathrm{GaN}$ of $2.5 \mu \mathrm{m}$ thickness, and a $\mathrm{GaN}$ buffer layer. The carrier concentration of the sample is $7.4 \times 10^{12} \mathrm{~cm}^{-2}$ and the electron mobility is $2500 \mathrm{~cm}^{2} / \mathrm{V} \mathrm{s}$ at $4.3 \mathrm{~K}$. The sample was placed inside a $6 \mathrm{~T}$ Oxford superconducting magnet, and was immersed in liquid helium. Raising the temperature is done by a balance between the controlled heating and the injected liquid helium. For the microwave-modulated $\mathrm{SdH}$ measurements, the microwave was generated by Gigatronics GT 9000 $\mathrm{S}$ microwave sweeper and guided to the sample surface by a microwave coaxial cable. The oscillatory resistivity was measured by a conventional lock-in amplifier with a reference frequency provided by a function generator modulating the microwave output.

It is known that the longitudinal resistivity $\rho_{x x}$ in the $\mathrm{SdH}$ oscillation is given by $[3,4]$

$\rho_{x x}=\rho_{0}\left[1+4 D_{T}(X) \exp \left(-\pi / \omega_{c} \tau_{q}\right)\right]$.

We know the temperature-dependent term is $D_{T}(X)=$ $X / \sinh X$, where $X=2 \pi^{2} k_{\mathrm{B}} T / \hbar \omega_{c}$. Therefore, the amplitude of the $\mathrm{SdH}$ oscillations is given by

$\Delta \rho_{x x}=4 \rho_{0} D_{T}(X) \exp \left(-\pi / \omega_{c} \tau_{q}\right)$

that depends on the carrier temperature-dependent factor $D_{T}(X)$. We can easily find that $D_{T}(X)$ decreases progressively with increasing temperature $T$. We thus observe the reduction of the $\mathrm{SdH}$ oscillatory amplitude under the illumination of continuous microwave. Our observation can be attributed to the effect of carrier heating $[5,6]$. The free carriers near the Fermi level absorb the incident microwave and become hot carriers. When considering the thermal energy with background temperature $(4.3 \mathrm{~K})$ in liquid helium, that provides about $0.4 \mathrm{meV}$ smaller than the band gap energy of $\mathrm{GaN}(\approx 3.5 \mathrm{eV})$. However, the frequency of the microwave is $300 \mathrm{MHz}-300 \mathrm{GHz}$, we have electric energy $E=h v \approx 10^{-3} \mathrm{meV}$. Therefore, we provide the energy to the carriers but do not excite electrons in the valance band. These hot carriers possess an equivalent temperature that may be higher than the lattice temperature, since the sample is immersed in liquid helium at $4.3 \mathrm{~K}$. Hence the $\mathrm{SdH}$ amplitude decreases compared with that obtained without microwave radiation.

When we consider the microwave-modulated $\mathrm{SdH}$ oscillations, as shown in Fig. 1. Let us see the difference between $\rho_{x x}$ with and without microwave illumination, which is

$$
\begin{aligned}
& \rho_{x x}(T)-\rho_{x x}(T+\Delta T) \\
& \quad=4 \rho_{0} \exp \left(\frac{-\pi}{\omega_{c} \tau_{q}}\right)\left[D_{T}(X)-D_{T+\Delta T}(X)\right] .
\end{aligned}
$$

Then the amplitude of the SdH oscillation depends sensitivity on the temperature-dependent factor $D_{T}(X)-D_{T+\Delta T}(X)$, that is an increasing function with increasing $\Delta T$. Now we have an enhanced amplitude of the SdH oscillations, as shown in Fig. 1(b). Therefore, the sensitivity of the $\mathrm{SdH}$ measurement can be improved. 


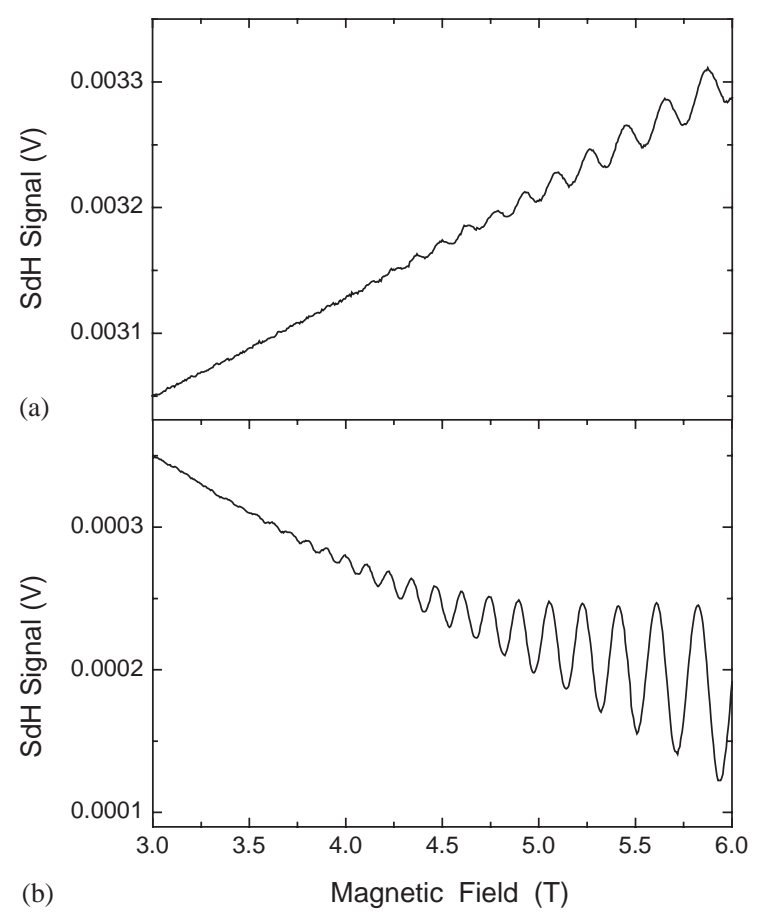

Fig. 1. (a) The conventional $\mathrm{SdH}$ oscillations on the $\mathrm{GaN} / \mathrm{Al}_{0.4} \mathrm{Ga}_{0.6} \mathrm{~N}$ heterostructure taken at a background temperature of $4.3 \mathrm{~K}$; (b) the microwave-modulated $\mathrm{SdH}$ pattern under the modulation of a $3 \mathrm{GHz}$ microwave radiation at the same temperature of $4.3 \mathrm{~K}$.

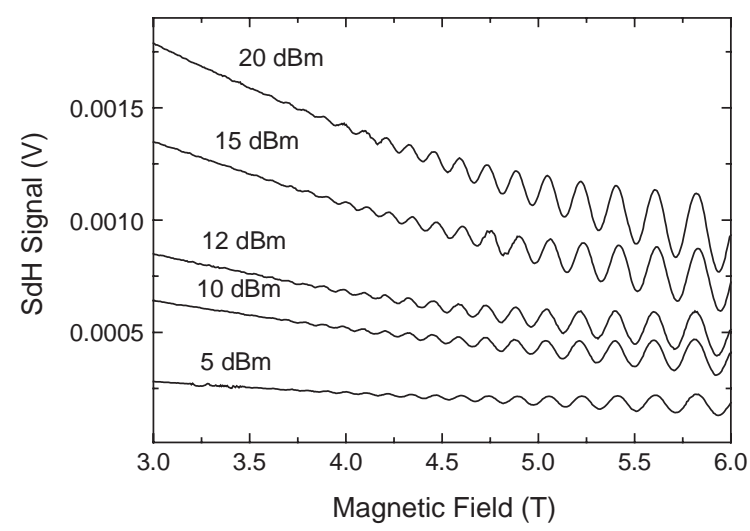

Fig. 2. The dependence of microwave-modulated SdH measurements on microwave power at temperature $4.3 \mathrm{~K}$. The microwave frequency is $4 \mathrm{GHz}$.

Fig. 2 shows the dependence of the $\mathrm{SdH}$ pattern on microwave power $(5-20 \mathrm{dBm})$. It is evident that the $\mathrm{SdH}$ amplitude increases with increasing microwave

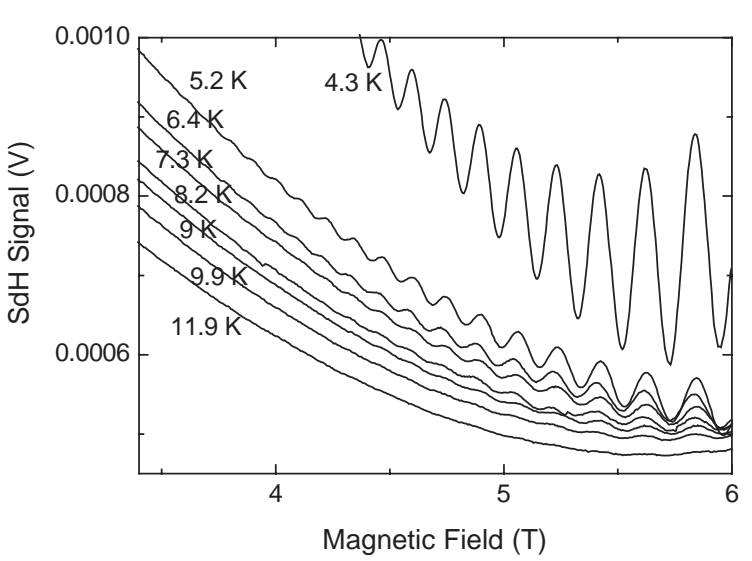

Fig. 3. The dependence of microwave-modulated SdH measurements on temperature with microwave frequency $3.7 \mathrm{GHz}$ and microwave power $20 \mathrm{dBm}$.

power. This result can also be interpreted in terms of the electron heating effect from microwave absorption. The amount of electron heating is increased as microwave power is increased. Therefore, increasing microwave power brings about higher carrier-equivalent temperatures. The higher the electron temperatures during illumination, the larger the difference between the oscillatory $\mathrm{SdH}$ amplitude. Thus, the microwave-modulated $\mathrm{SdH}$ pattern is enhanced.

We also increase directly the temperature of the sample to see the effect of microwave-modulated $\mathrm{SdH}$ oscillations. Fig. 3 shows the dependence of microwave-modulated $\mathrm{SdH}$ measurements on temperature. We see that the $\mathrm{SdH}$ amplitudes decrease as the sample temperature increases. At a fixed microwave power, an increase in the lattice temperature decreases the difference $D_{T}(X)-D_{T+\Delta T}(X)$, resulting in a smaller SdH amplitude. However, the SdH oscillatory signal can be observed at a temperature up to about $10 \mathrm{~K}$. Therefore, the observation of SdH oscillations at this relatively high temperature clearly demonstrates the usefulness of the microwave-modulated $\mathrm{SdH}$ measurement.

Let us now turn our attention to the second part of this paper. We investigate the transport properties of a gated GaN electron system. By changing the applied gate voltage, we are able to vary the electron density in our system. Fig. 4 shows the four-terminal longitudinal magnetoresistivity as a function of perpendicular magnetic field at various gate voltages. In 


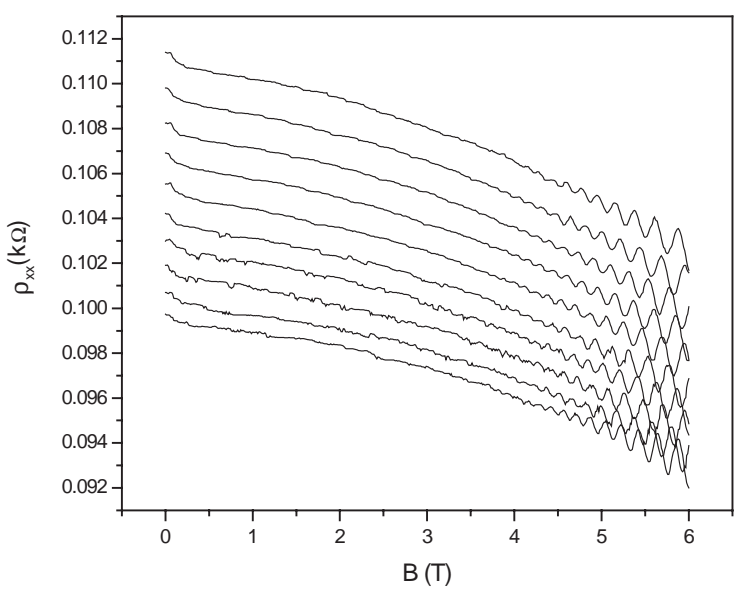

Fig. 4. Four-terminal magnetoresistivity measurements at different gate voltages. From top to bottom: $V_{\mathrm{g}}=+0.6$ to $-0.3 \mathrm{~V}$ in $0.1 \mathrm{~V}$ steps.

all cases, the carrier densities determined from the period of the $\mathrm{SdH}$ oscillations and those measured from the classical Hall effect at low fields are within 2.8\% difference. These results, together with the fact that we only observe a single series of $\mathrm{SdH}$ oscillations, show that there is only one $2 \mathrm{D}$ subband occupied in the GaN quantum well.

By changing the applied gate voltage $\left(V_{\mathrm{g}}\right)$ from -3.5 to $+0.5 \mathrm{~V}$, we can vary the electron density from $3.11 \times 10^{12}$ to $6.95 \times 10^{12} \mathrm{~cm}^{-2}$ in our system. We now use a simple parallel-plate capacitor model in which one plate of the capacitor is the metallic surface gate whilst the other is the 2DEG. Our simple model allows us to estimate the averaged distance between the 2DEG and the GaN/AlGaN interface. Fig. 5 shows the electron density as a function of $V_{\mathrm{g}}$. We can see that the electron density shows a linear dependence on $V_{\mathrm{g}}$. From the linear fit the depth of the 2DEG is estimated to be $640 \AA$ below the surface. This is somewhat larger than the as-grown thickness of the AlGaN layer $400 \AA$. Our simple model allows us to estimate the averaged distance between the 2DEG and the GaN/AlGaN interface to be $240 \AA$. This is not unexpected since in a triangular quantum well, the maximum of the electron wave function distribution is at a certain distance away from the semiconductor interface.

Let us now describe the carrier dependence of mobility in our system. For a fixed gate voltage, we are able to measure the electron density from the period

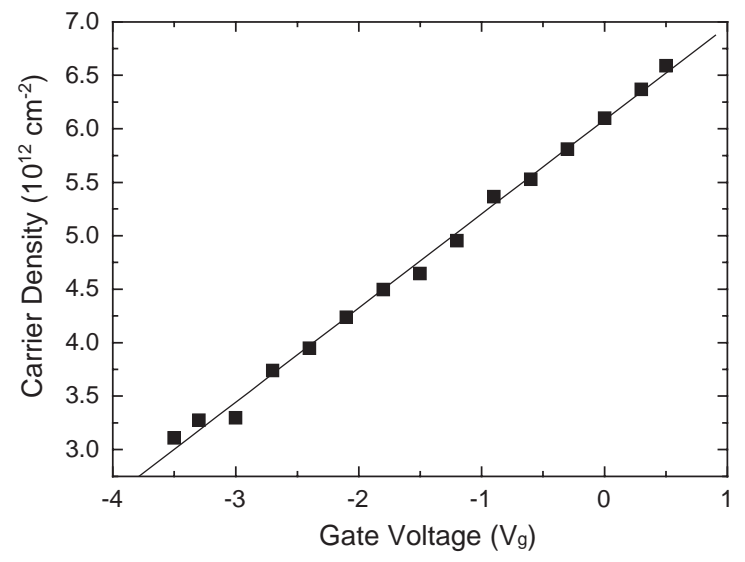

Fig. 5. The electron density versus gate voltage at $T=4.3 \mathrm{~K}$.

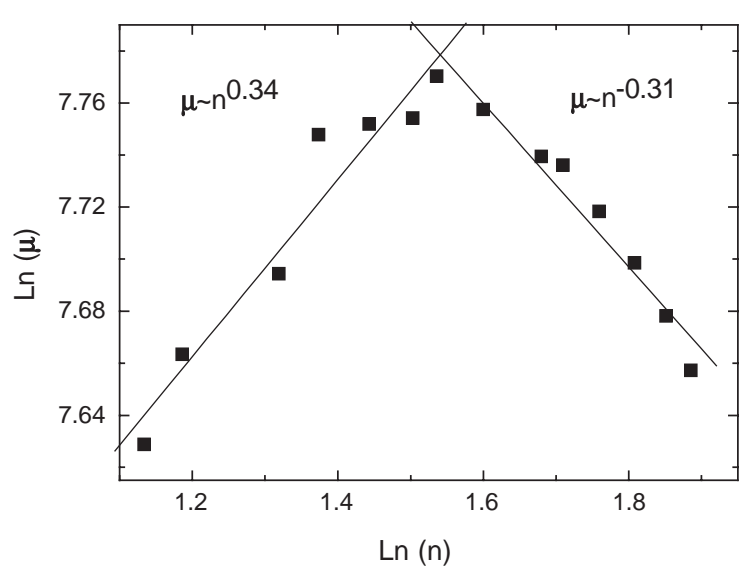

Fig. 6. The logarithm of mobility $\ln (\mu)$ as a function of the logarithm of electron density $\ln (n)$.

of the SdH oscillations. From the relation $\sigma=n \mathrm{e} \mu$ where $\sigma$ is the zero-field conductivity, we can readily calculate the mobility $\mu$. By repeating this process, we can calculate the mobility $\mu$ at different electron density $n$. In order to elucidate the underlying physics of the mobility dependence on electron density, we plot $\ln (\mu)$ as a function of $\ln (n)$, as shown in Fig. 6. In our system at higher electron concentrations $(n>4.65 \times$ $10^{12} \mathrm{~cm}^{-2}$ ) the measured electron mobility is found to be a decreasing function of electron concentration $\mu \sim n^{-0.31}$. The decreasing mobility with increasing concentration is consistent with interface roughness scattering $[7,8]$. The effect of interface roughness 
is expected to decrease mobility with increasing electron concentration as the electron wave function is pressed closely against the GaN/AlGaN heterointerface. At lower electron densities $\left(n<4.65 \times 10^{12}\right.$ $\mathrm{cm}^{-2}$ ), the measured electron mobility is found to be an increasing function of electron concentration as $\mu \sim n^{0.34}$. This is consistent with remote ionized impurity scattering $[9,10]$ in GaN/AlGaN electron systems. This behavior is well known and is typical of $\mathrm{GaAs} / \mathrm{AlGaAs}$ heterostructures. In this regime, mobility increases with density as $\mu \sim n^{\alpha}$ where $\alpha$ typically varies from 0.7 to $1.5[7,11]$. At a low temperature of $4.3 \mathrm{~K}$, the major contribution to electron mobility comes from temperature-independent scattering processes such as remote ionized-impurity scattering and interface roughness scattering. At low electron densities, the contribution from interface roughness scattering can be expected to be very small. On the other hand, as the electron density in the quantum well increases, the potentials of the remote ionized impurities are screened more effectively by the electron gas. Therefore, the electron mobility will increase. The measured electron mobility is found to be an increasing function of electron density as $\mu \sim n^{0.34}$. A possible reason for the smaller exponent 0.34 is that the mobility in our system is approximately five times lower than those reported for a similar carrier density in a $\mathrm{GaN}$ electron system $[7,10]$.

In conclusion, we have presented conventional and microwave-modulated Shubnikov-de Haas ( $\mathrm{SdH}$ ) measurements on $\mathrm{GaN}$ electron systems. By using the microwave-modulation technique, we are able to drastically enhance the sensitivity of $\mathrm{SdH}$ measurements. According to the studies of the dependence on microwave power and lattice temperature, we attribute the mechanism of the enhancement to the hot electron effect induced by microwave absorption. This technique has the advantage of keeping the carrier density fixed and is very useful for wide-band-gap heterostructures in which moderate mobilities and heavier effective mass (rapid damping $\mathrm{SdH}$ amplitudes) are common. We have also investigated the transport properties of a gated GaN electron system. By changing the applied gate voltage, we are able to vary the carrier concentration so as to study the mobility dependence on electron density. At higher electron concentrations $\left(n>4.65 \times 10^{12} \mathrm{~cm}^{-2}\right)$ the measured electron mobility is found to be a decreasing function of electron concentration $\mu \sim n^{-0.31}$. The decreasing mobility with increasing concentration is consistent with interface roughness scattering. At lower electron densities $\left(n<4.65 \times 10^{12} \mathrm{~cm}^{-2}\right)$, the measured electron mobility is found to be an increasing function of electron concentration as $\mu \sim n^{0.34}$. This is consistent with remote ionized impurity scattering, though the measured exponent 0.34 is smaller than the typical value (0.7-1.5) observed in an $\mathrm{AlGaN} / \mathrm{GaN}$ electron system. A possible reason is that our sample mobility is approximately five times lower than those in other devices for a similar electron density.

\section{Acknowledgements}

This work was funded by the NSC, Taiwan, and the MOE programme for Promoting Academic Excellence of University (89-N-FA01-2-4-3). Gil-Ho Kim was supported by National R\&D Project for Nano Science and Technology (Contract No. M1-0212-04-0003) of MOST.

\section{References}

[1] S.E. Schacham, E.J. Haugland, S.A. Alterovitz, Appl. Phys. Lett. 61 (1992) 551

[2] H. Linke, B. Kowalski, P. Ramvall, P. Emanuelsson, P. Omling, K. Oettinger, M. Drechsler, B.K. Meyer, Appl. Phys. Lett. 62 (1993) 2725.

[3] A. Isihara, L. Smrcka, J. Phys. C.: Solid State Phys. 19 (1986) 6777.

[4] P.T. Coleridge, R. Stoner, R. Fletcher, Phys. Rev. B 39 (1988) 1120.

[5] H.Y. Fan, in: R.K. Willardson, A.C. Beer (Eds.), Semiconductors and Semimetals, Vol. 3, Academic Press, New York, 1967, p. 405.

[6] J.C. Fan, Y.H. Chang, Y.F. Chen, J.F. Whang, F.F. Fang, W.J. Tsai, C.Y. Chang, China J. Phys. 35 (1997) 917.

[7] M.J. Manfra, L.N. Pfeiffer, K.W. West, H.L. Stormer, K.W. Baldwin, J.W.P. Hsu, D.V. Lang, R.J. Molner, Appl. Phys. Lett. 77 (2000) 2888.

[8] B.R. Nag, S. Mukhopadhyay, M. Das, J. Appl. Phys. 86 (1999) 459

[9] W. Walukiewicz, H.E. Ruda, J. Lagowski, H.C. Gatos, Phys. Rev. B 29 (1984) 4818.

[10] L. Hsu, W. Walukiewicz, Phys. Rev. B 56 (1997) 1520.

[11] T. Ando, J. Phys. Soc. Japan 51 (1982) 3900. 